Ann. Biol. anim. Bioch. Biophys., I97I, 11 (3), 399-4I3.

\title{
ÉQUIPEMENT ENZYMATIQUE DU PANCREAS EXOCRINE CHEZ LE LAPIN, MISE EN PLACE ET EVOLUTION DE LA NAISSANCE AU SEVRAGE. RELATION AVEG LA COMPOSITION DU RÉGIME ALIMENTAIRE
}

\author{
F. LEBAS, T. CORRING et D. COURTOT $\left({ }^{(}\right)$ \\ avec la collaboration technique de Anne-Marie Guevgneav, G. SARdi et Y. Cotta \\ Station de Recherches sur l'Elevage des Porcs, \\ Centre national de Recherches zootechniques, I. N. R. A., \\ 78 - Jouy-en-Josas \\ Département de Biologie, \\ Institut national des Sciences appliquées, \\ 69 - Villeurbanne
}

\section{RÉSUMÉ}

L'étude a porté sur r 20 lapereaux de race Californienne de la naissance au $32^{\mathrm{e}}$ jour d'âge. Le poids vif, les quantités de lait et d'aliment complémentaire ingérées ont été régulièrement contrôlées au niveau de chaque portée. Il a été procédé, à différents âges, sur broyat de pancréas entier à la détermination de la teneur en protéines totales de la glande et des activités enzymatiques de l'amylase, la lipase, la trypsine et la chymotrypsine.

Les résultats montrent que la vitesse de croissance du pancréas est inférieure à celle de l’organisme jusqu'à l'âge de ${ }_{4} 4$ jours et lui devient égale par la suite.

Les diverses enzymes étudiées sont présentes dès la naissance et l'on assiste à une très forte augmentation des activités totales de la lipase, l'amylase et la chymotrypsine à partir du $24^{\mathrm{e}}$ jour d'âge, tandis que l'activité trypsique totale demeure constante durant toute la période étudiée.

La comparaison de ces variations à celles du régime alimentaire laisse supposer que la biosynthèse de l'amylase est fortement stimulée par l'alimentation, celle du chymotrypsinogène l'est à un degré moindre tandis que celle du trypsinogène reste indifférente. La lipase enfin, montre un comportement particulier qu'il est difficile d'expliquer et les variations de son activité semblent indépendantes de la composition du régime et notamment de sa teneur en lipides.

\section{INTRODUCTION}

On sait que les proportions respectives des enzymes du pancréas exocrine varient beaucoup d'une espèce animale à l'autre. Celle de l'amylase, par exemple, est faible chez le bœuf, moyenne chez le Porc et le Chien, forte chez le Rat. Chaque espèce

(1) Adresse actuelle : Laboratoire de Pharmacologie-Toxicologie, I. N. R. A., I8o, Chemin de Tournefeuille, $3 I$ - Toulouse. 
semble donc organiser la biosynthèse de ses enzymes pancréatiques selon un plan qui lui est particulier. Au sein d'une même espèce la synthèse des différentes enzymes varie au cours du temps principalement pendant le jeune âge, comme l'ont montré Walker (I959), Hartman et al. (I96I) sur le Porcelet, Corring et Aumaitre (I970 a) sur le Raton. Les travaux sur ce sujet restent malheureusement rares.

D'autre part au cours de la période d'allaitement, et au moment du sevrage, le régime alimentaire du jeune est profondément modifié. On est tenté de rapprocher ces variations alimentaires de celles des enzymes digestives d'origine pancréatique. Il s'agit en fait de reprendre dans le cas du jeune, l'hypothèse d'une adaptation des enzymes pancréatiques formulée au cours des travaux déjà anciens de PAVLOV (I9I3) et précisés depuis par Grossman et al. (I942) par REBOUD et al. (I962). Un travail sur le jeune permet de plus une étude du phénomène dans des conditions normales d'alimentation. En effet les variations de composition du régime alimentaire, provoquées par les auteurs précités, sont très brutales, la teneur en protéines (caséine) passant par exemple de 5 à 70 p. Ioo dans l'alimentation de rats en croissance.

Au cours du travail présenté nous avons donc tenté d'aborder la mise en place et l'évolution de 1'équipement enzymatique du pancréas de lapin, espèce peu étudiée, de la naissance au sevrage. Nous avons de plus cherché à utiliser la variation naturelle réalisée chez le lapereau dont le régime passe de $50 \mathrm{p}$. Ioo de protéines à $15-20 \mathrm{p}$. Ioo par exemple au moment du sevrage (PRUD'HON et BEL, I968; CowIE, I969 ; LEBAS, I $970 \mathrm{a}$ ) pour tester l'hypothèse de l'adaptation au régime dans des conditions normales d'élevage.

\section{MATÉRIEL ET MÉTHODES}

\section{A. - Les animaux}

L'expérience a porté sur I 20 lapereaux de race Californienne nés le même jour et abattus en Io stades répartis au cours d'une période d'allaitement de 30 jours ou juste après le sevrage. Les conditions de logement et d'alimentation des lapines comme de leurs portées ont déjà été décrites par l'un d'entre nous (LeBas, I968).

Tous les lapereaux ont été identifiés à la naissance et leur ordre d'abattage tiré au sort au sein de chaque portée. Sauf pour le premier et le dernier stade, un lapereau du même âge a été introduit en remplacement des sujets abattus de manière à maintenir constant l'effectif de la portée à 8 indiviđus.

Un aliment complémentaire du lait et riche en amidon est mis à la disposition des lapereaux dès le $15^{\mathrm{e}}$ jour. Les consommations d'aliment sont contrôlées chaque jour par différence entre les quantités distribuées et les refus. Les consommations de lait sont estimées par la perte de poids de la lapine au cours de la tétée quotidienne (LeBas, I968 et 1969).

Les portées expérimentales y compris les animaux introduits au titre de remplacement sont pesées globalement avant chaque prélèvement pour analyses.

\section{B. - Abattages et prélèvements}

Des essais préalables destinés à préciser les dates d'abattages ont montré une variation dans le développement du pancréas exocrine vers le $\mathrm{I}_{4} \mathrm{e}$ jour après la mise bas. Aussi les animaux ont-ils été abattus à l'âge de I, 7, 9, II, I 4, I7, 2I, 24, 30 (sevrage) et 32 jours. Les lapereaux ne tétant qu'une seule fois par 24 heures, les prélèvements d'animaux ont été réalisés à chaque stade avant la tétée quotidienne dans chacune des I 2 portées expérimentales sauf pour le $32^{\mathrm{e}}$ jour où les lapereaux sont prélevés au sein de 2 portées supplémentaires. Les animaux prélevés sous la mère ont été pesés individuellement puis décapités entre 9 heures et ro heures du matin, soit 26 heures en moyenne après la dernière tétée. 
Un échantillon de lait de 40 à $80 \mathrm{~g}$ a été prélevé mécaniquement (LEBAs, I970 $b$ ) à chaque stade sur 6 des 12 lapines en expérience, afin de déterminer la composition chimique de l'ingéré des lapereaux. Un échantillonnage moyen a également été réalisé sur l'aliment complémentaire aux fins d'analyse.

$$
\text { C. - Dosages }
$$

Immédiatement après abattage, le pancréas est extrait, pesé frais et homogénéisé à l'aide d'un ultra-turrax Staufen type TP I 8/2 dans de l'eau distillée glacée. Après homogénéisation des pancréas, il est procédé sur le broyat à la mesure de la teneur du tissu en protéines totales, à la détermination des activités de la chymotrypsine, trypsine, amylase et lipase. L'activation du chymotrypsinogène et du trypsinogène est effectuée avec de la trypsine Sigma (2 X) à raison de Io $\mathrm{mg}$ et $5 \mathrm{mg}$ respectivement pour roo $\mathrm{mg}$ de protéines totales pancréatiques. Les méthodes utilisées sont décrites dans un précédent travail (CoRRING et AUMAITRE, I970). Cependant, nous en rappellerons brièvement le principe. Les activités trypsique et chymotrypsique sont déterminées par titrimétrie après hydrolyse de substrat spécifique, respectivement du benzo- $l$-arginineéthyl-ester (BAEE) et de l'acétyl-l-tyrosine-éthyl-ester (ATEE). Les activités amylasique et lipasique sont déterminées respectivement après hydrolyse de l'amidon et par titrimétrie après action de l'enzyme sur une huile d'olive standard.

Les échantillons de lait sont analysés en vue de la détermination de la matière sèche (à $60^{\circ} \mathrm{C}$ sous vide) des cendres (12 heures à $55^{\circ} \mathrm{C}$ ) des protéines totales (Kjeldhal $\mathbf{N} \times 6,3^{8}$ ) du lactose (méthode de SomogyI). Les matières grasses sont estimées par différence. Un échantillon moyen d'aliment a été utilisé pour la détermination de la teneur en protéines, en matières grasses, en cendres, en cellulose, de la matière sèche.

\section{RÉSULTATS}

\section{A. - Évolution pondérale}

La croissance pondérale des lapereaux est linéaire durant les 3 premières semaines puis elle s'accélère entre 20 et 30 jours d'âge (tabl. I, fig. I). Le pancréas suit un développement comparable. Cependant, l'étude de l'évolution du logarithme du poids du

\section{TABLEAU I}

Variations du poids vif des lapereaux, du poids total du pancreas frais et de la quantité des protéines totales pancréatiques entre les 3 premières semaines et la $4^{\mathrm{e}}$ semaine d'âge

\begin{tabular}{l|c|c|c}
\hline & Variation journalière & $\begin{array}{c}\text { Variation journalière } \\
\text { relative } \\
\text { Période } 2 / \text { Période 1 } \\
(\%)\end{array}$ \\
\hline Pancréas (mg) ........ & $\begin{array}{c}\text { 1-21 jours } \\
\text { Période } 1 \\
\text { Période } 2\end{array}$ \\
\hline Protéines totales (mg) ... & 16,3 & 53,5 & 328 \\
\hline Poids vif (g) ......... & 3,5 & 11,2 & 320 \\
\hline \hline
\end{tabular}

pancréas frais $(Y)$ en fonction de celui du poids vif $(X)$ permet de définir 2 droites successives au cours du temps (fig. 2).

$$
\begin{aligned}
& Y_{1}=0,70 X_{1}+I, 87 \text { entre } I \text { et } I 4 \text { jours } \\
& Y_{2}=1,07 X_{2}+0,112 \text { entre } I_{7} \text { et } 30 \text { jours }
\end{aligned}
$$


Le développement relatif du pancréas, de la naissance au $30^{\circ}$ jour, est marqué par un changement d'allométrie, le coefficient passant de 0,70 à $I, 07$. En comparant les deux pentes à la pente théorique : I, on peut préciser que le pancréas passe d'une phase d'allométrie minorante $(a=0,70 \mathrm{P} \leqslant 0,0 \mathrm{I})$ à une phase d'isométrie

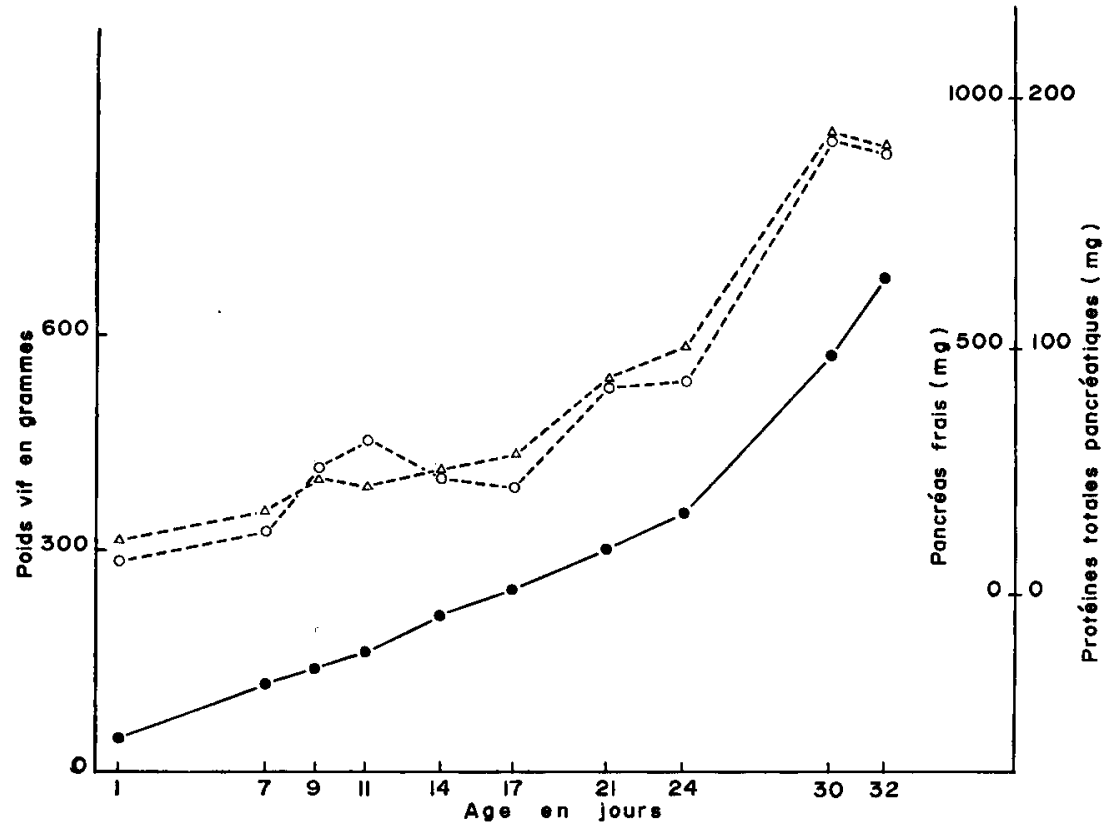

FIG. I. - Evolution moyenne du poids vif, -- du poids frais de pancréas $\Delta---\cdots$ et de la quantité de proteines totales pancréatiques $0-----0$ chez le Lapin de la naissance au sevrage

$(a=\mathrm{I}, 07 \mathrm{NS})$, lorsque le poids vif se situe entre $\mathrm{I} 65$ et $265 \mathrm{~g}$. Cette zone correspond approximativement à des lapereaux au début de leur troisième semaine. Les lapereaux présentent donc une croissance accélérée dès la fin de la $3^{\mathrm{e}}$ semaine, le phénomène étant plus marqué pour le pancréas que pour le poids vif.

\section{B. - Composition des aliments}

La composition chimique du lait n'est pas stable au cours du temps (tab1. 2). Après la phase colostrale on assiste à une relative constance des teneurs pour les différents éléments durant 3 semaines environ. Entre 2 I et 24 jours, par contre, le lait s'enrichit en matières sèches, en protéines, en matières grasses et en cendres tandis que la teneur en lactose diminue, ce dernier tendant à disparaître vers le $30^{\mathrm{e}}$ jour. Il faut également remarquer que l'extractif non azoté principalement composé d'amidon représente 45 p. Ioo de l'aliment complémentaire du lait.

\section{C. - Quantités consommées}

La consommation de lait présente un maximum en fin de troisième semaine (tabl. 3), au moment même où les lapereaux commencent à consommer l'aliment 


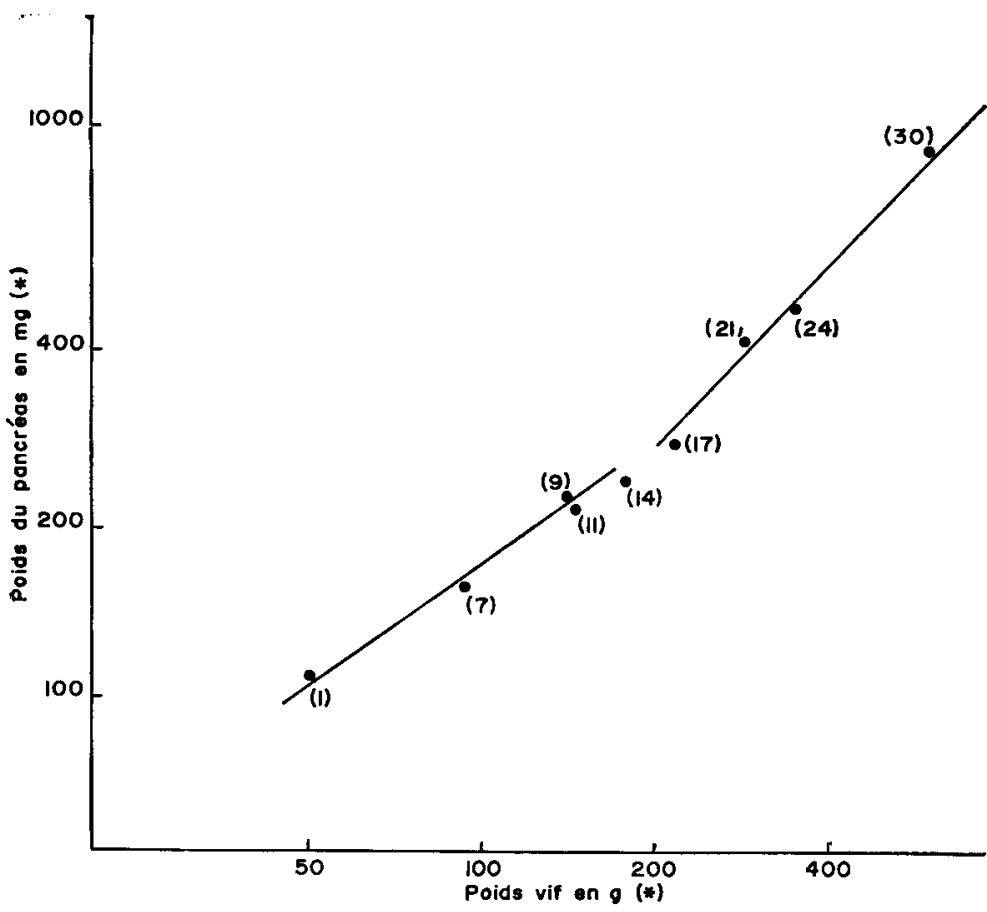

* Echelle logarithmique

Fig. 2. - Evolution pondérale du pancréas frais

en fonction du poids vif chez le Lapin de la naissance au seurage

(chiffres entre parenthèses correspondent aux differents stades d'abattage, en jours)

TABLEAU 2

Composition du lait et de l'aliment complémentaire

\begin{tabular}{|c|c|c|c|c|c|c|c|c|c|c|}
\hline \multirow{2}{*}{$\begin{array}{l}\text { Composants en } \% \\
\text { de la matière } \\
\text { fraîche } \pm s_{\bar{x}}\end{array}$} & \multicolumn{9}{|c|}{$\begin{array}{l}\text { Laits aux différents stades } \\
\text { (en jours) }\end{array}$} & \multirow{2}{*}{$\begin{array}{l}\text { Aliment } \\
\text { comple- } \\
\text { mentaire }\end{array}$} \\
\hline & 1 & 7 & 9 & 11 & 14 & 17 & 21 & 24 & 30 & \\
\hline Matière sèche & $\begin{array}{c}31,4 \\
\pm \quad 0,6\end{array}$ & $\begin{array}{c}25,6 \\
\pm \quad 0,6\end{array}$ & $\begin{array}{c}26,2 \\
\pm \quad 0,7\end{array}$ & $\begin{array}{r}25,7 \\
\pm \quad 0,9\end{array}$ & $\begin{array}{c}25,6 \\
\pm \quad 0,5\end{array}$ & $\begin{array}{c}26,6 \\
\pm \quad 0,9\end{array}$ & $\begin{array}{c}26,7 \\
\pm \quad 1,0\end{array}$ & $\begin{array}{c}33,4 \\
\pm \quad 2,4\end{array}$ & $\begin{array}{c}37,4 \\
\pm \quad 2,3\end{array}$ & 90,6 \\
\hline Protéines & $\begin{array}{c}13,5 \\
\pm \quad 0,3\end{array}$ & $\begin{array}{c}13,6 \\
\pm \quad 0,4\end{array}$ & $\begin{array}{c}12,8 \\
\pm \quad 0,2\end{array}$ & $\begin{array}{c}13,6 \\
\pm \quad 0,3\end{array}$ & $\begin{array}{c}13,4 \\
\pm \quad 0,5\end{array}$ & $\begin{array}{c}13,0 \\
\pm \quad 0,6\end{array}$ & $\begin{array}{c}13,1 \\
\pm \quad 0,2\end{array}$ & $\begin{array}{c}15,5 \\
\pm \quad 0,3\end{array}$ & $\begin{array}{c}16,9 \\
\pm \quad 0,9\end{array}$ & 23,0 \\
\hline Matières grasses & $\begin{array}{c}14,7 \\
\pm \quad 0,6\end{array}$ & $\begin{array}{c}9,1 \\
\pm \quad 0,6\end{array}$ & $\begin{array}{c}10,4 \\
\pm \quad 0,6\end{array}$ & $\begin{array}{c}8,9 \\
\pm \quad 0,8\end{array}$ & $\begin{array}{c}9,0 \\
\pm \quad 0,3\end{array}$ & $\begin{array}{c}10,0 \\
\pm \quad 0,6\end{array}$ & $\begin{array}{c}10,3 \\
\pm \quad 1,0\end{array}$ & $\begin{array}{c}14,4 \\
\pm 1,8\end{array}$ & $\begin{array}{c}17,5 \\
\pm 1,4\end{array}$ & 2,3 \\
\hline Cendres & $\begin{array}{c}1.65 \\
\pm \quad 0,06\end{array}$ & $\begin{array}{c}2,03 \\
\pm \quad 0,06\end{array}$ & $\begin{array}{c}1,98 \\
\pm \quad 0,05\end{array}$ & $\begin{array}{c}2,18 \\
\pm \quad 0,03\end{array}$ & $\begin{array}{r}2,25 \\
\pm \quad 0,08\end{array}$ & $\begin{array}{c}2,57 \\
\pm \quad 0,25\end{array}$ & $\begin{array}{c}2,36 \\
\pm \quad 0,06\end{array}$ & $\begin{array}{c}2,61 \\
\pm \quad 0,11\end{array}$ & $\begin{array}{r}2,78 \\
\pm \quad 0,10\end{array}$ & 9,3 \\
\hline Lactose & $\begin{array}{c}1,63 \\
\pm \quad 0,03\end{array}$ & $\begin{array}{c}0,95 \\
\pm \quad 0,04\end{array}$ & $\begin{array}{c}1,09 \\
\pm \quad 0,02\end{array}$ & $\begin{array}{c}1,02 \\
\pm \quad 0,04\end{array}$ & $\begin{array}{c}0,99 \\
\pm \quad 0,06\end{array}$ & $\begin{array}{c}1,04 \\
\pm \quad 0,04\end{array}$ & $\begin{array}{c}0,95 \\
\pm \quad 0,06\end{array}$ & $\left\{\begin{array}{c}0,80 \\
\pm \quad 0, \mathbf{1 1}\end{array}\right.$ & $\begin{array}{c}0,20 \\
\pm \quad 0,13\end{array}$ & - \\
\hline Cellulose & - & - & 一 & 一 & 一 & 一 & 一 & - & 一 & 10,6 \\
\hline
\end{tabular}


complémentaire. L'ingestion de ce đernier croît d'une manière extrêmement rapide jusqu'à 32 jours.

TABLEAU 3

Consommations journalières de lait et d'aliment complémentaire par portée (Effectif 8) aux différents stades

\begin{tabular}{c|c|c|c|c|c|c|c|c|c|c}
\hline \hline $\begin{array}{c}\text { Quantités ingérées } \\
\text { en } \mathrm{g} \pm \mathrm{s} \bar{x}\end{array}$ & 1 & 7 & 9 & 11 & 14 & 17 & 21 & 24 & 30 & 32 \\
\hline Lait & $\begin{array}{c}60 \\
\pm 7\end{array}$ & $\begin{array}{c}172 \\
\pm 12\end{array}$ & $\begin{array}{c}148 \\
\pm 10\end{array}$ & $\begin{array}{c}180 \\
\pm 10\end{array}$ & $\begin{array}{c}186 \\
\pm 9\end{array}$ & $\begin{array}{c}215 \\
\pm 10\end{array}$ & $\begin{array}{c}206 \\
\pm 9\end{array}$ & $\begin{array}{c}169 \\
\pm 15\end{array}$ & $\begin{array}{c}155 \\
\pm 20\end{array}$ & - \\
\hline $\begin{array}{c}\text { Aliment } \\
\text { complémentaire }\end{array}$ & - & - & - & - & - & $\begin{array}{c}0,3 \\
31\end{array}$ & $\begin{array}{c}113 \\
\pm 07\end{array}$ & $\begin{array}{c}339 \\
\pm 1,1\end{array}$ \\
\hline \hline
\end{tabular}

A partir de la mesure des quantités de lait et d'aliment complémentaire ingérées ainsi que de leurs éléments constitutifs, nous avons calculé la composition de la ration moyenne des lapereaux (fig. 3). Il nous a également été possible de suivre l'évolution des quantités ingérées de matière sèche, de protéines et de lipides (fig. 4). Grâce à la mesure du poids des animaux nous avons estimé la consommation des trois nutriments précédents par unité de poids vif (fig. 5).

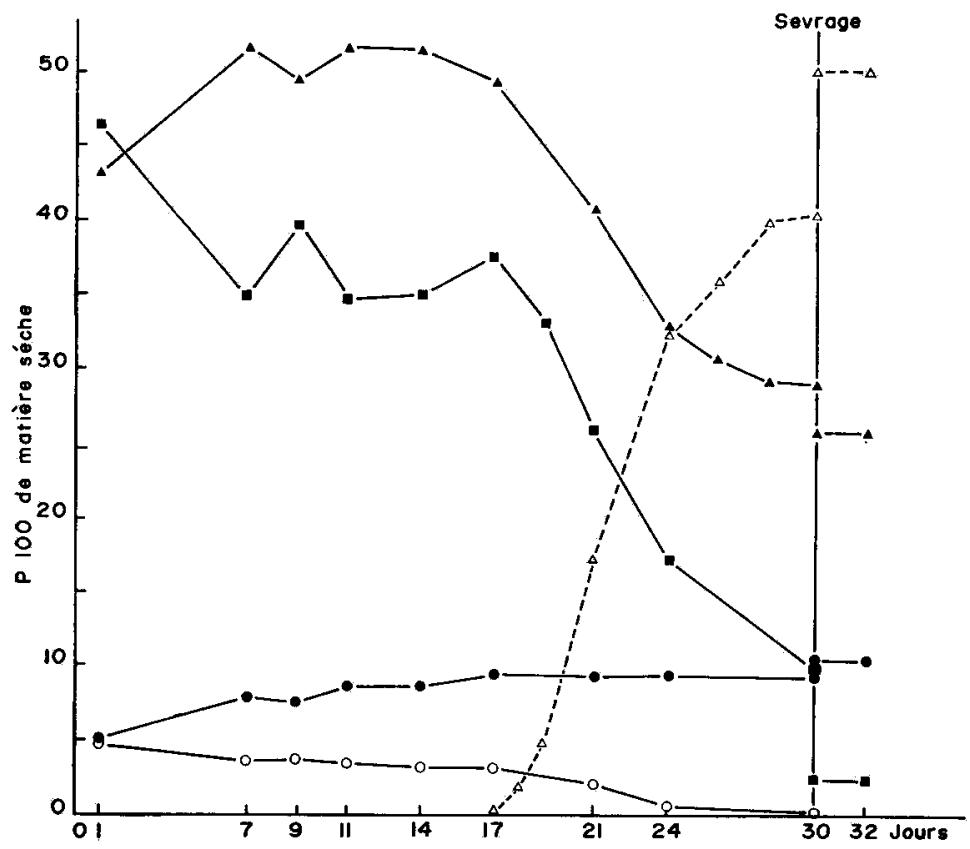

FIG. 3. - Composition de la matière sèche moyenne ingérée par les lapereaux de la naissance au sevrage

protéines brutes - matières grasses

- cendres $0-0$ lactose $\Delta---\Delta$ Extractif non azoté 


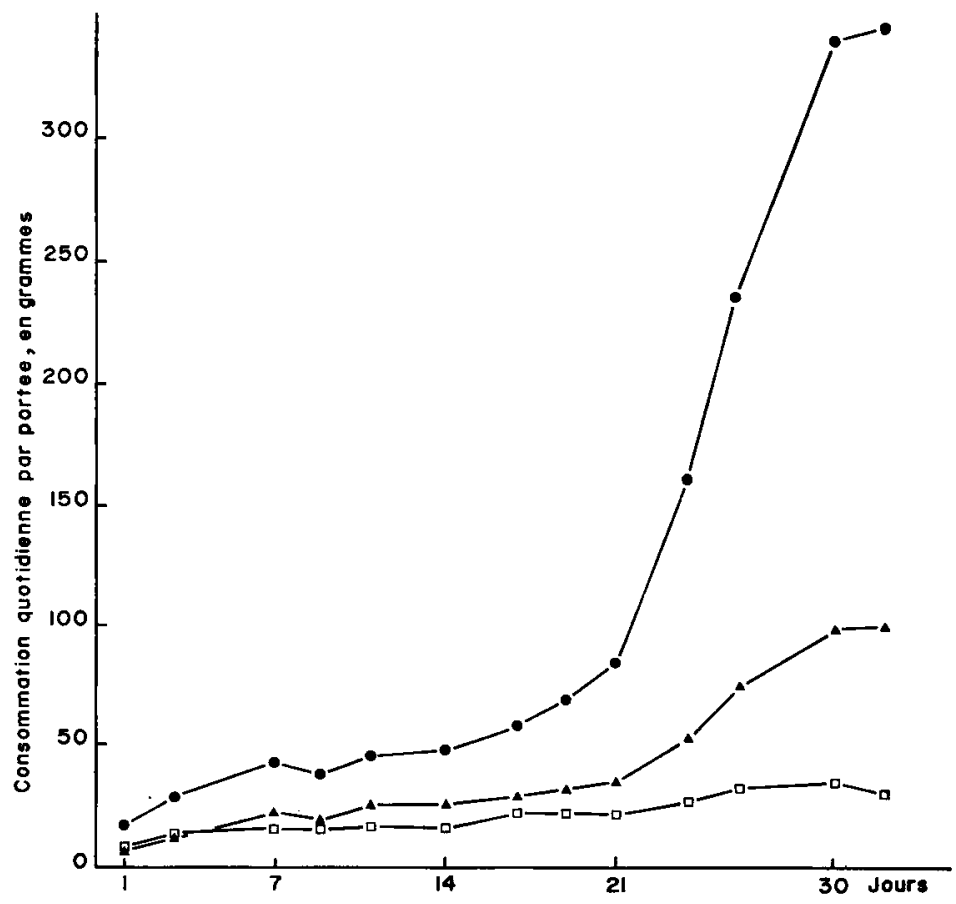

FIG. 4. - Évolution des quantités ingérées moyennes de matière sèche - de protéines $\Delta \longrightarrow \wedge$ de matières grasses $\square \longrightarrow \square$ par portée de 8 lapereaux au cours de la période d'allaitement

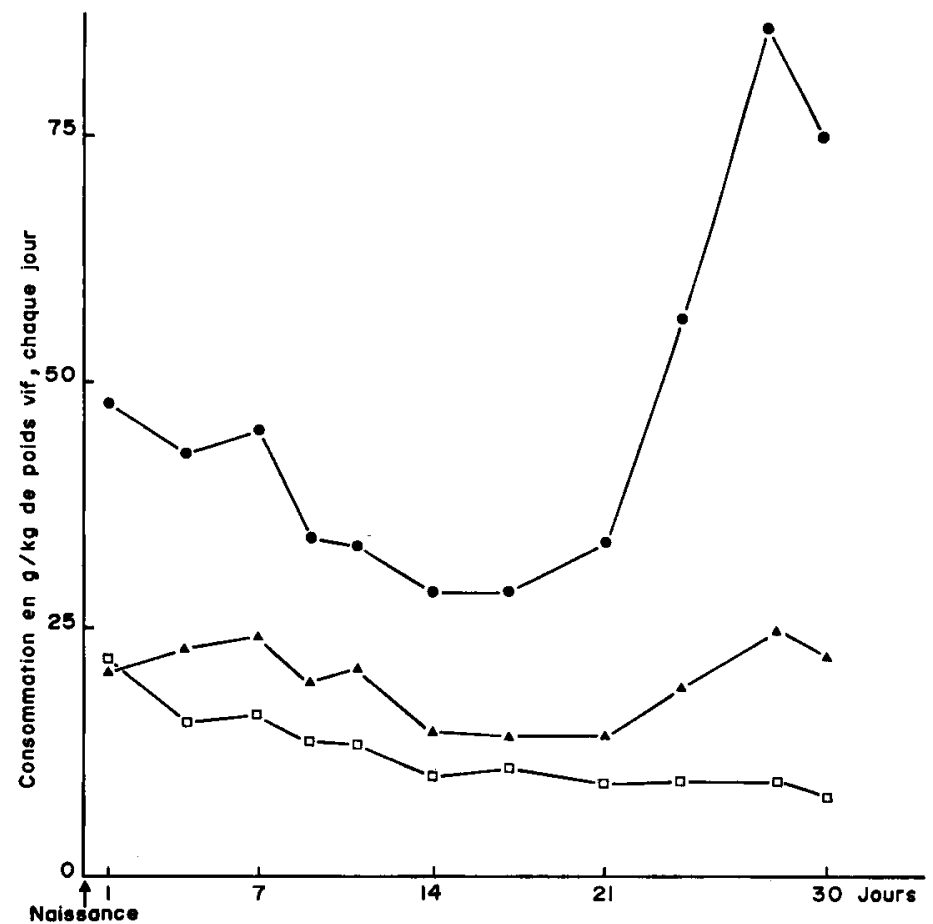

FIG. 5. - Évolution des quantités ingéres par unité de poids vif en matière sèche $\bullet-\bullet$, en proteines $\triangle-\perp$ et en matieres grasses $\square-\square$ pour des lapereaux sous la mère 
L'étude de ces différentes courbes montre une diminution de la teneur en pro téines, en lipides et en lactose de la ration moyenne (fig. 3) dès le début de la consommation d'aliment complémentaire ( $I 7$ jours). Ceci correspond à l'apparition dans le régime des animaux d'une proportion croissante de granulé complémentaire riche en amidon et en cellulose. Par contre, la quantité de protéines ingérées augmente alors rapidement suivant en cela l'évolution de l'ingestion de matière sèche. Ce phénomène est plus net pour l'apport par unité de poids vif (fig. 5) que pour les quantités absolues (fig. 4). La quantité de matières grasses ingérées chaque jour suit une évolution plus régulière : légère augmentation pour la consommation absolue, diminution constante pour la quantité par unité de poids vif.

On assiste donc au cours de l'allaitement à une réduction de la teneur en protéines de la ration qui passe de 50 à $25 \mathrm{p}$. Ioo de la matière sèche en une douzaine de jours. Parallèlement les quantités consommées de protéines, comme celles d'amidon, augmentent, qu'on les exprime globalement ou par unité de poids vif.

\section{D. - Activités enzymatiques du pancréas}

\section{Activités enzymatiques totales.}

L'évolution des activités enzymatiques étudiées montre une variation présentant deux phases distinctes (fig. 6 et 7 ). Ainsi il apparaît une tendance à l'augmentation, faible mais régulière, de la lipase et de l'amylase jusqu'à 1a fin de la $3^{\mathrm{e}}$ semaine et une augmentation très importante par la suite. L'activité lipasique est en effet multipliée par 4 entre la naissance et 20 jours, par 28 entre la naissance et 30 jours. L'activité amylasique est multipliée par 5 et 30 pendant les mêmes périodes.

En ce qui concerne les enzymes protéolytiques, seule l'activité chymotrypsique suit une évolution semblable aux deux précédentes enzymes. En effet, elle est multipliée par $r, 4$ entre la naissance et 20 jours et par Io entre la naissance et 30 jours. L'activité trypsique, par contre demeure constante pendant toute l'étude.

On assiste donc pour 3 des 4 enzymes étudiées à un démarrage, puis à une accélération de l'activité totale à partir du $20^{\mathrm{e}}$ jour environ.

\section{Activités enzymatiques spécifiques.}

Lorsque l'on considère les diverses activités spécifiques exprimées en fonction du poids frais de l'organe, ou par rapport aux protéines totales (tabl. 4), il apparait une différence de comportement entre la lipase et l'amylase d'une part et les enzymes protéolytiques d'autre part. Nous retrouvons ainsi, pour les activités lipasique et amylolytique une évolution identique à celle observée avec les activités totales. Par contre, l'activité spécifique de la trypsine décroît régulièrement de la naissance au $3^{\mathrm{e}}$ jour, alors que l'activité spécifique de la chymotrypsine décroît entre la naissance et le II $^{\mathrm{e}}$ jour puis demeure sensiblement constante par la suite.

L'étude de l'évolution du rapport chymotrypsine/trypsine (tabl. 4) montre que le niveau du chymotrypsinogène dans le pancréas d'abord sensiblement égal à celui du trypsinogène, lui devient supérieur, le rapport passant de $I$ à 2 au cours de la $4^{\text {e }}$ semaine d'âge. 


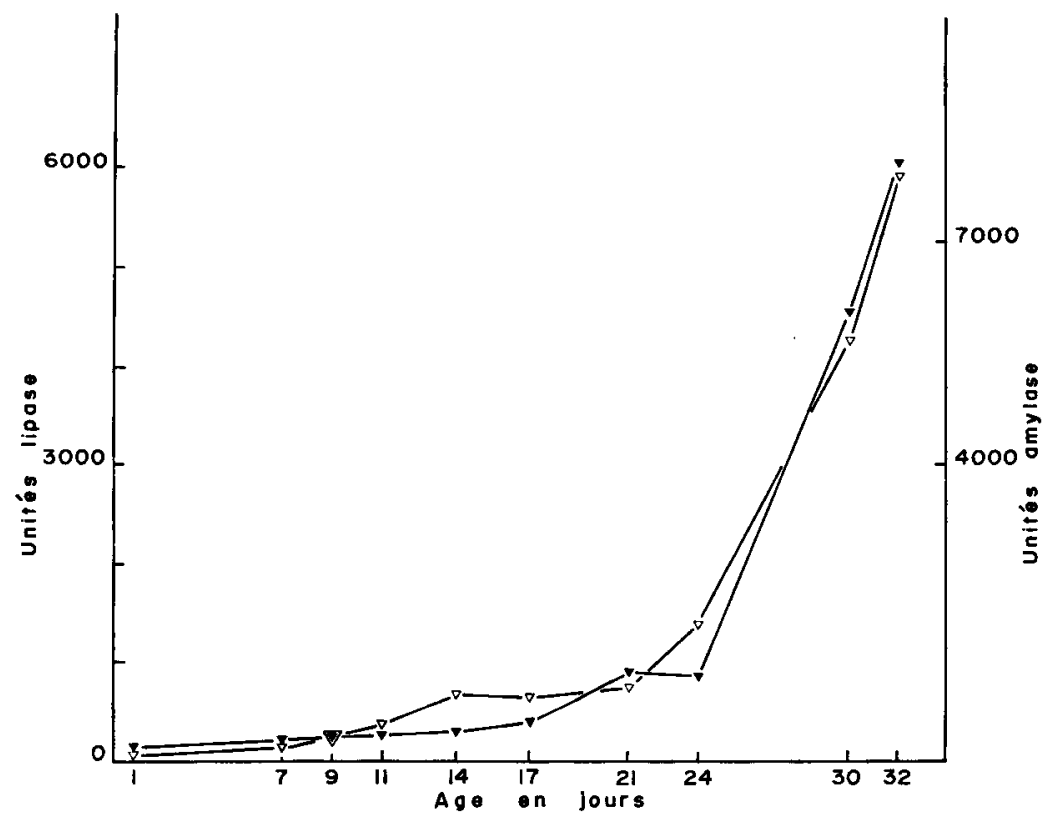

FIG. 6. - Activités enzymatiques totales par pancréas de l'amylase $\triangle \longrightarrow \triangle$ et de la lipase $\Delta-\Lambda$ chez le Lapin de la naissance au sevrage

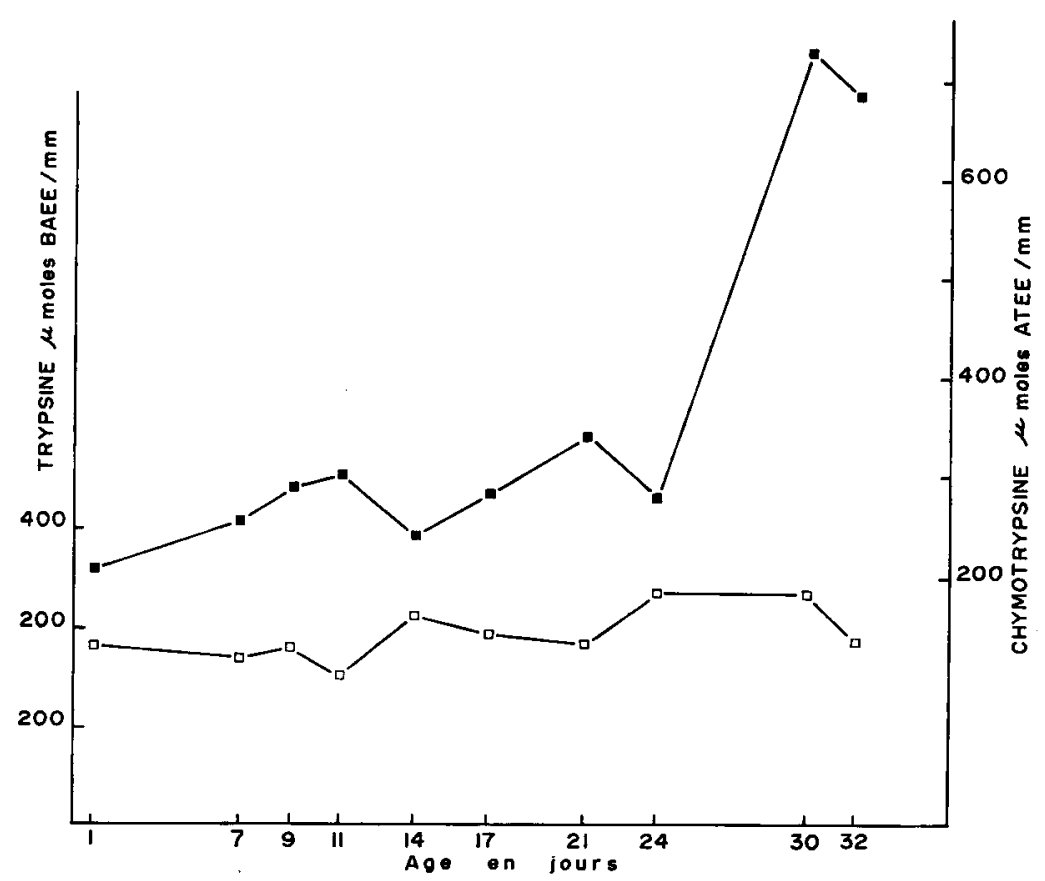

FIG. 7. - Activités enzymatiques totales par pancreas de la trypsine $\square-\square$ et de la chymotrypsine $\square$ chez le Lapereau de la naissance au sevrage 
TABLEAU 4

Activités spécifiques de la chymotrypsine, trypsine, lipase et amylase dans le pancréas des lapeveaux de la naissance au sevrage

\begin{tabular}{|c|c|c|c|c|c|c|c|c|c|c|}
\hline \multirow{2}{*}{ Moyennes $\pm \overrightarrow{x_{x}}$} & \multicolumn{10}{|c|}{ Stades } \\
\hline & 1 & 7 & 9 & 11 & 14 & 17 & 21 & 24 & 30 & 32 \\
\hline $\begin{array}{l}\text { Protéines totales } \\
\text { (mg) }\end{array}$ & $\begin{array}{l}14,6 \\
\pm 1,3\end{array}$ & $\begin{array}{l}23,4 \\
\pm 1,9\end{array}$ & $\begin{array}{l}49,8 \\
\pm 4,4\end{array}$ & $\begin{array}{c}61,6 \\
\pm 3,4\end{array}$ & $\begin{array}{l}48,4 \\
\pm 3,8\end{array}$ & $\begin{array}{l}41,4 \\
\pm 3,2\end{array}$ & $\begin{array}{l}83,5 \\
\pm 7,5\end{array}$ & $\begin{array}{l}84,9 \\
\pm 4,1\end{array}$ & $\begin{array}{c}184,1 \\
\pm \quad 10,1\end{array}$ & $\begin{array}{l}177,0 \\
\pm 7,6\end{array}$ \\
\hline $\begin{array}{l}\text { Chymotrypsine par } \\
\text { mg de protéines }\left({ }^{(}\right)\end{array}$ & $\begin{array}{l}20,9 \\
\pm 1,8\end{array}$ & $\begin{array}{c}11,2 \\
\pm 0,5\end{array}$ & $\begin{array}{c}6,1 \\
\pm 0,5\end{array}$ & $\begin{array}{c}4,9 \\
\pm 0,3\end{array}$ & $\begin{array}{r}5,2 \\
\pm 0,5\end{array}$ & $\begin{array}{c}6,7 \\
\pm 0,2\end{array}$ & $\begin{array}{c}4,1 \\
\pm 0,4\end{array}$ & $\begin{array}{c}3,3 \\
\pm 0,2\end{array}$ & $\begin{array}{c}3,9 \\
\pm 0,2\end{array}$ & $\begin{array}{c}3,9 \\
\pm 0,2\end{array}$ \\
\hline $\begin{array}{c}\text { Trypsine }\left({ }^{2}\right) \\
\text { par mg de protéines }\end{array}$ & $\begin{array}{l}14,2 \\
\pm 0,8\end{array}$ & \begin{tabular}{|l|}
12,3 \\
$\pm 0,9$
\end{tabular} & $\begin{array}{r}6,2 \\
\pm \quad 0,5\end{array}$ & $\begin{array}{c}3,7 \\
\pm \quad 0,2\end{array}$ & $\begin{array}{c}6,9 \\
\pm 0,6\end{array}$ & $\begin{array}{l}7,7 \\
\pm 0,7\end{array}$ & $\begin{array}{c}3,4 \\
\pm \quad 0,3\end{array}$ & $\begin{array}{c}4,1 \\
\pm \quad 0,2\end{array}$ & $\begin{array}{c}1,9 \\
\pm 0,1\end{array}$ & $\begin{array}{c}1,7 \\
\pm 0,1\end{array}$ \\
\hline $\begin{array}{l}\text { Rapport Chymo- } \\
\text { trypsine/Trypsine }\end{array}$ & 1,47 & 0,90 & 0,99 & 1,33 & 0,75 & 0,87 & 1,18 & 0,80 & 2,12 & 2,29 \\
\hline $\begin{array}{c}\text { Lipase } / \mathrm{mg}\left({ }^{3}\right) \\
\text { proteines }\end{array}$ & $\begin{array}{c}6,2 \\
\pm 0,4 \\
\end{array}$ & $\begin{array}{r}9,6 \\
\pm 0,6 \\
\end{array}$ & $\begin{array}{r}5,2 \\
\pm \quad 0,3 \\
\end{array}$ & $\begin{array}{c}5,0 \\
\pm 0,4 \\
\end{array}$ & $\begin{array}{c}6,7 \\
\pm 0,9 \\
\end{array}$ & $\begin{array}{c}9,5 \\
\pm 0,6\end{array}$ & $\begin{array}{l}11,4 \\
\pm 1,2\end{array}$ & $\begin{array}{c}10,1 \\
\pm 0,8\end{array}$ & $\begin{array}{l}24,7 \\
\pm 2,5\end{array}$ & $\begin{array}{l}33,1 \\
\pm 3,0\end{array}$ \\
\hline $\begin{array}{c}\text { Amylase/mg }\left({ }^{4}\right) \\
\text { protéines }\end{array}$ & $\begin{array}{c}10,0 \\
\pm 1,2\end{array}$ & $\begin{array}{c}11,4 \\
\pm 1,1\end{array}$ & $\begin{aligned} & 6,7 \\
\pm & 1,1\end{aligned}$ & $\begin{array}{c}7,0 \\
\pm 1,5\end{array}$ & $\begin{array}{l}19,5 \\
\pm 5,0\end{array}$ & $\begin{array}{l}20,1 \\
\pm 3,3\end{array}$ & $\begin{array}{l}10,9 \\
\pm 1,2\end{array}$ & $\begin{array}{l}21,0 \\
\pm 3,5\end{array}$ & $\begin{array}{l}31,9 \\
\pm 1,9\end{array}$ & $\begin{array}{l}45,6 \\
\pm 2,3\end{array}$ \\
\hline
\end{tabular}

(1) $\mu$ mole ATEE/mn.

(2) $\mu$ mole BAEE/mn.

(3) $\mu$ mole acide gras $/ \mathrm{mn}$.

(4) Nombre extrémités réductrices apparues après une hydrolyse de $20 \mathrm{mn}$ de l'amidon.

\section{DISCUSSION}

La traite de la moitié des lapines pour l'obtention des échantillons de lait aurait pu introduire une variation supplémentaire dans la croissance et le développement des jeunes. L'analyse statistique n'a permis de déceler de différence significative pour aucun des critères de la présente étude ; aussi avons-nous réalisé les calculs et fourni tous les résultats moyens pour l'ensemble des lapereaux issus des I2 portées que leur mère ait été traite ou non.

La croissance pondérale des lapereaux est comparable à celle enregistrée par VENGE (1963), SANTORO (I967) et LEBAS (r969). La production 1aitière des lapines est analogue à celle mentionnée par LEBAS en I968. La composition chimique du lait suit une évolution proche de celle enregistrée par CowIE (I969). Le départ très rapide de la consommation d'aliment complémentaire à partir du $\mathrm{I} 7^{\mathrm{e}}$ jour est un phénomène remarquable qui avait déjà été mentionné par LEBAS (I970 a). Ainsi les différentes manipulations nécessitées par l'expérimentation n'ont pas altéré les performances des animatx. 


\section{A. - Développement relatif du pancréas}

Le développement pondéral du pancréas se traduit par le passage d'une phase d'allométrie minorante à une phase d'isométrie. Ce résultat est à rapprocher des observations histologiques de CoURTOT et al. (I970). En effet, à l'aide de la technique d'immuno-fluorescence, la détection des enzymes protéolytiques dans les cellules pancréatiques du lapereau permet aux auteurs de conclure que les cellules observées ne deviennent identiques à celles de l'adulte qu'aux environs $d u{ }^{1} 4^{e}$ jour date du changement du coefficient d'allométrie.

De même, il apparaît à cette période une réaction de fluorescence dans la lumière des acini, ce qui indique le début de l'excrétion enzymatique, donc le fonctionnement effectif du pancréas dans son rôle de glande digestive.

\section{B. - Activités enzymatiques et régime alimentaire}

\section{Activités enzymatiques totales et consommations par lapereau.}

On constate parallèlement au phénomène du développement pondéral de la glande pancréatique, et au début de la consommation d'aliment complémentaire une augmentation du niveau des principales enzymes : lipase, amylase, chymotrypsine. L'activité trypsique seule ne varie pas. La différence entre ces deux enzymes protéolytiques ne doit pas surprendre; en effet d'autres résultats mettent en évidence le comportement semble-t-il indépendant, de ces deux enzymes et notamment la grande faculté d'adaptation de la chymotrypsine à toute stimulation pancréatique. Une telle propriété avait déjà été soulignée à l'occasion de travaux portant sur le taux de protéines dans la ration du rat, par BEN ABDELJLII et DESNUELLE (I964), REBOUD et al. (I966) et sur la nature de celles-ci, par SNOoK et MEYER ((I964) chez le rat, PEKAS et al. (I964) chez le porcelet. De même cette faculté d'adaptation de la chymotrypsine a été montrée chez le Rat en croissance (Corring et AUMAITRE, I970 b) au cours d'une étude sur l'effet du traitement thermique du tourteau de soja. Ainsi donc nous constatons un parallélisme entre l'augmentation de l'ingéré (hydrates de carbone, protéines et lipides) et le développement du pancréas qui se traduit par une augmentation du potentiel enzymatique total.

\section{Activités enzymatiques spécifiques et consommation relative.}

De nombreux résultats d'expérience désormais classiques démontrent une adaptation des enzymes pancréatiques à une variation de la composition du régime alimentaire (REBOUd et al., r962; MARChis-MouREN et al., 1963; BEN ABDELJLIL, et DESNUELLE, 1964). C'est ainsi que selon ces auteurs :

- l'activité chymotrypsique varie dans le même sens que la teneur en protéines du régime alors que l'activité trypsique varie très peu;

- 1'activité amylasique suit la teneur du régime en amidon ;

- l'activité lipasique semble indépendante de la teneur en matières grasses.

a) Les activités protéolytiques.

Durant les deux premières semaines de vie des lapereaux, les activités spécifiques de la trypsine et de la chymotrypsine, élevées à la naissance décroissent régulière- 
ment. Pendant cette même période la teneur en protéines du lait, seul aliment, est relativement stable tandis que diminue la quantité ingérée par unité de poids vif.

En fin d'allaitement 1'activité trypsique spécifique continue à décroître tandis que l'activité chymotrypsique se stabilise. Simultanément la teneur en protéines de la ration moyenne diminue rapidement alors que la quantité de protéines ingérée par unité de poids vif augmente.

Comme les auteurs précités, nous trouvons donc une évolution de l'activité spécifique de la trypsine indépendante de celle de la chymotrypsine ou des protéines alimentaires. Mais contrairement à leurs résultats nous observons une évolution opposée de l'activité chymotrypsique spécifique et de la teneur en protéines du régime. Dans notre cas il n'y aurait pas adaptation de la chymotrypsine à la teneur en protéines du régime mais à la quantité de protéines ingérée globale ou mieux à la quantité de protéines ingérée par unité de poids vif.

On peut penser qu'à la naissance, le pancréas dispose d'un " stock " de molécules de trypsinogène et de chymotrypsinogène. Par suite, malgré l'augmentation de la quantité totale des protéines pancréatiques et le développement pondéral de la glande, ce potentiel de précurseurs protéolytiques ne varie pas en ce qui concerne le trypsinogène, d'où la diminution de l'activité spécifique, les enzymes protéolytiques ne jouant qu'un rôle faible dans la digestion du lait. Lors de la consommation de l'aliment complémentaire, la stimulation de la croissance globale du pancréas éventuellement induite par la modification du régime alimentaire serait suffisante pour obtenir le taux optimum d'enzymes protéolytiques, en particulier de chymotrypsinogène. Cette hypothèse est en accord avec des résultats comparables trouvés chez le jeune rat par CORRING et AUMAITRE (I970 a), sachant toutefois que dans ces observations, la biosynthèse des deux précurseurs protéolytiques reste absolument parallèle.

\section{b) Les activités amylasique et lipasique.}

Durant la première partie de l'allaitement et tant que le lait reste le seul aliment, l'activité spécifique de l'amylase augmente régulièrement, mais faiblement. Dès que le lapereau consomme de l'aliment complémentaire donc dès l'apparition d'amidon dans la ration (teneur et quantité ingérée) l'activité de l'amylase s'accroît rapidement.

L'activité amylasique suit donc 1'accroissement de la teneur en amidon dans le régime, mais également 1'augmentation concomitante des quantités ingérées (brutes ou par unité de poids).

Ces résultats sont donc en accord avec les données classiques obtenues par d'autres auteurs, sur de nombreuses autres espèces animales.

Si l'on considère maintenant l'activité spécifique de la lipase dans le pancréas du lapereau, nous obtenons une évolution semblable à celle de l'amylase. Durant la première phase de l'allaitement, l'activité lipasique augmente légèrement tandis que la teneur en lipides est stable, que les quantités ingérées totales augmentent et que les quantités ingérées par unité de poids vif diminuent. Au cours de la seconde moitié de 1'allaitement, 1'activité spécifique de la lipase augmente rapidement alors que la teneur en lipides du régime, comme la quantité de lipides ingérée par unité de poids vif, diminuent et que seule la quantité totale de lipides ingérée par le lapereau augmente.

Contrairement aux résultats de DFSNUELLE et al. (I962) sur le Rat après sevrage 
nous observons donc sur le lapereau une variation importante de l'activité spécifique de la lipase. Ce phénomène, semble-t-il propre au lapin, pourrait être lié à l'augmentation des quantités de lipides ingérées mais il reste certainement, dans nos conditions, indépendant de la teneur en lipides du régime ou de la quantité de lipides ingérée par unité de poids vif.

En résumé, les résultats obtenus ne sont pas exactement comparables aux observations classiques sur l'adaptation du pancréas à la composition du régime, effectuées chez le Rat. Mais il faut noter d'une part que les espèces étudiées sont différentes et d'autre part que les conditions expérimentales divergent sur un point fondamental : la nature du régime alimentaire. En effet, la modification du régime alimentaire à partir $\mathrm{du} \mathrm{I} \mathrm{I}^{\mathrm{e}}$ jour est fort complexe, car à une augmentation de la quantité ingérée vient s'ajouter un changement de la nature des divers constituants du régime.

Il est difficile à la suite de ce travail de déterminer les causes exactes des variations observées. Est-ce la consommation d'aliment solide qui induit, soit par effet mécanique, soit par effet biochimique, le développement de l'activité fonctionnelle pancréatique, ou le développement de cette activité (sous l'effet de 1'âge par exemple) entraîne-t-il la prise et la consommation d'aliment solide? Signalons qu'en culture organotypique, isolée de tout stimulus alimentaire, l'activité de synthèse demeure très réduite, ce qui ferait plutôt admettre la première hypothèse. L'alimentation activerait et modulerait la biosynthèse des protéines enzymatiques par le pancréas, le relai entre 1'alimentation et 1'activité pancréatique restant très complexe. Les travaux de Prochazka et al. (I964) et Yalowski et al. (I969) laissent supposer que les substances corticoïdes seraient impliquées dans ce relai.

\section{CONCLUSION}

L'ensemble de nos résultats nous conduit à souligner les particularités de la sécrétion enzymatique du pancréas du lapin placé dans les conditions naturelles de sevrage. Le pancréas du lapereau de 3 semaines réagit aux stimuli alimentaires par une augmentation de son activité enzymatique. Il y aurait une adaptation aux variations quantitatives plus que qualitatives du régime alimentaire.

La biosynthèse de l'amylase est stimulée fortement, celle du chymotrypsinogène l'est à un degré moindre, tandis que celle du trypsinogène est indifférente. L,a lipase a un comportement particulier qui reste difficile à expliquer et ses variations semblent bien indépendantes de la composition du régime et notamment de la teneur en lipides.

Des expérimentations complémentaires (sevrage total de plus en plus précoce, élevage des lapereaux jusqu'au $30^{e}$ jour d'âge nourris uniquement du lait de lapine) nous permettront de savoir s'il s'agit réellement d'une adaptation au régime alimentaire ou d'une évolution en fonction de l'âge (ou du poids) des lapereaux. 


\title{
SUMMARY
}

\author{
FORMATION AND DEVELOPMENT OF THE ENZYME APPARATUS \\ OF THE EXOCRINE PANCREAS OF YOUNG RABBITS FROM BIRTH TO WEANING \\ INFLUENCE OF THE COMPOSITION OF THE DIET
}

The current investigation was carried out on 120 young Caifornian rabbits from birth to 32 days of age. Live weights and amounts of milk and supplemental feed ingested were recorded for each litter. The rate of total protein and the enzyme activity of amylase, lipase, trypsin and chymotrypsin were estimated from samples of whole pancreas at various ages.

Growth rate of the pancreas was found to be lower than that of the animal up to day ${ }_{4} 4$ and parallel to it after that age.

The various investigated enzymes were present from birth. A marked increase in lipase, amylase and chymotrypsin total activities was observed from day 24 on, whereas trypsin total activity remained constant throughout the range of the experiment.

A comparison of these variations with dietary changes inclined us to the view that the feed greatly stimulates amylase biosynthesis, chymotrypsinogen biosynthesis to a lesser extent, and is ineffectual on trypsinogen. The variations of lipase were rather peculiar and seem to be irrespective of the diet and its lipid constituents.

\section{RÉFÉRENCES BIBIIOGRAPHIQUES}

Ben Abdeljlil A., Desnuelle P., rg64. Sur l'adaptation des enzymes exocrines du pancréas à la composition du régime. Biochim. Biophys. Acta, 81, I36-149.

Corring T., Aumaitre A., I970 $a$. Mise en place et évolution de l'équipement enzymatique du pancréas exocrine du jeune rat pendant la période embryonnaire, l'allaitement et le sevrage. Ann. Biol. anim. Bioch. Biophys., 10, 43-44.

Corring T., Aumaitre A., I970 $b$. Effet du traitement thermique du tourteau de soja sur la variation de l'équipement enzymatique du pancréas exocrine et les performances pondérales du rat après seyrage. Ann. Biol. anim. Bioch. Biophys., 10, 443-457.

CowiE A. T., I969. Variations in the yield and the composition of the milk during lactation in the rabbit and the galactopoietic effect of prolactin. J. Endocr., 44, 437-450.

Courtot D., Corring T., Laviolette P., i97o. Détection des proenzymes protéolytiques du pancréas du Lapin par immunofluorescence (embryon, nouveau-né, adulte). Ann. Biol. anim. Bioch. Biophys., 10, $29 x-299$.

Desnuelle P., Reboud J.-P., Ben Abdeljlil A., I962. Influence of the composition of the diet on the enzyme content of rat pancreas., in Ciba Foundation Symposium of the exocrine pancreas ; Churchill (London), p. 90 .

Grossman M. I., Greengard H., Ivy A. C., r942. The effect of dietary composition on pancreatic enzymes. Amer. J. Physiol., 138, 676-682.

Hartman P. A., Hays V. W., Baker R. C., Neagle L. H., Catron D. W., ig6r. Digestive enzyme development in the young pig. J. Anim. Sci, 20, II4-I23.

Lebas F., I968. Mesure qualitative de la production laitière chez la Lapine. Ann.Zootech., 17, I68-182.

Lebas F., I969. Alimentation lactée et croissance pondérale du Lapin avant sevrage. Ann. Zootech., 18, $197-208$.

Lebas F., I970 a. Alimentation et croissance des lapereaux sous la mère. Rec. Med. Vet., 146, 10651070 .

Lebas F., r97o b. Description d'une machine à traire les lapines. Ann. Zootech., 19, 223-228.

Marchis-Movren G., Pasero L., Desnuelle P., I963. Further studies on amylase biosynthesis by pancreas of rats fed on a starch-rich or a casein rich diet. Biochem. Biophys. Res. Comm., 13, 262-266.

Pavlov I. P., I9I3. The work of the digestive glands. Charles Griffin ed. London.

Peras J. C., Hays V. W., Thompson A. M., I964. Exclusion of the exocrine pancreatic secretion : effect on digestibility of soybean and milk protein by baby pigs at various ages, J. Nutr., 82, 277-285.

PRUd'Hon M., BeL L., I968. Le sevrage précoce des lapereaux et la reproduction des lapines. Ann. Zootech., 17, 23-30. 
Prochazka P., Hahn P., Koldovsky D., Noh Ynek M., Rokos J., 1964. The activity of $\alpha$-amylase, 13, 288-29I.

Reboud J.-P., Ben Abdeljlil A., Desnuelle P., I962. Variation de la teneur en enzymes du pancréas de rat en fonction de la composition des régimes. Biochim. Biophys. Acta, 58, 326-337.

Reboud J.-P., Marchis-Mouren G., Pasero L., Cozzone A., Desnuelle P., ig66. Adaptation de la vitesse de biosynthèse de l'amylase pancréatique et du chymotrypsinogène à des régimes riches en amidon ou en protéines. Biochim. Biophys., Acta, 117, 351-367.

SANToRo I967. Ingagini su alcune carateristiche bio-zootecniche del coniglio allevato per la produzione della carne. Riv. Zootec. Agric. Vet., 5, I I9-г 3 I.

SNook J. T., Meyer J. H., r964. Responses of digestive enzymes to dietary protein. J. Nutr., 88, 408-4I3.

VENGE O., 1963. The influence of nursing behaviour and milk production on early growth in rabbits. Anim. Beh., 11, 500-506.

Walker D. M., I959. The development of the digestive enzymes of the young animal. II. Carbohydrase enzyme development in the young pig. J. Agric. Sci., 52, 357-37o.

Yalowski U., Zelikson R., KuLKa G., I969. The effect of hydrocortisone on the accumulation of amylase in embryonic chick pancreas. Febs. Letters., 2, 323-326. 